\title{
Medida da ansiedade e depressão em pacientes no pré-operatório de cirurgias eletivas
}

\author{
Measure of anxiety and depression in postoperative patients undergoing elective surgeries
}

Medidas de ansiedad y depresión en pacientes en preoperatorio de cirugías electivas

\begin{abstract}
Mariana Avelino dos Santos ${ }^{1}$, Lídia Aparecida Rossi ${ }^{2}$, Luciana Paiva ${ }^{3}$, Rosana Aparecida Spadoti Dantas ${ }^{4}$
\end{abstract} Daniele Alcalá Pompeo ${ }^{5}$, Elaine Cristina Bomfim Machado ${ }^{6}$

\section{RESUMO}

Os procedimentos cirúrgicos aliados à hospitalização representam uma ameaça ao doente e sua família, devido às alterações físicas, reações psicológicas e sociais. Este estudo foi realizado com o objetivo de avaliar a presença de sinais e sintomas de ansiedade e depressão e verificar associações das patologias com as variáveis sexo e idade, em pacientes no pré-operatório de cirurgias eletivas. Os dados foram coletados em um Hospital Universitário de Ribeirão Preto e a amostra foi composta por 100 sujeitos que responderam a Escala de Ansiedade e Depressão Hospitalar (HADS) e a um instrumento de dados sociodemográficos e clínicos, e informações relativas às condições do período perioperatório. Na avaliação de ansiedade e depressão, $40 \%$ dos indivíduos foram considerados casos de ansiedade e $22 \%$ dos indivíduos foram considerados casos de depressão. Encontrou-se relação estatisticamente significante dos níveis dessas patologias com as variáveis sexo masculino $(p=0,001)$, feminino $(p=0,000)$ e idade até 45 anos $(p=0,000)$.

Descritores: Ansiedade; Depressão; Cirurgia; Assistência Perioperatória.

\section{ABSTRACT}

Surgical procedures associated with hospitalization represent a threat to the patient and his/her family, due to physical alterations and psychological and social reactions. This study aimed at assessing the presence of signs and symptoms of anxiety and depression and at verifying associations of the pathologies with the variables gender and age in postoperative patients undergoing elective surgeries. Data were collected in a teaching hospital of Ribeirão Preto and the sample was comprised of 100 participants who completed the Hospital Anxiety and Depression Scale (HADS) and an instrument designed to collect clinical and sociodemographic data, as well as information regarding the particpants'conditions in the postoperative period. In the assessment of anxiety and depression, $40 \%$ of the subjects were considered to have anxiety and $22 \%$ of the subjects were considered to be suffering from depression. A statistically significant relationship was found between the levels of the pathologies and the variables male ( $\rho=0.001)$ female $(p=0.000)$ and age younger than 45 years $(p=0.000)$.

Descriptors: Anxiety; Depression; Surgery; Perioperative Care.

\section{RESUMEN}

Los procedimientos quirúrgicos sumados a la hospitalización representan una amenaza al enfermo y su familia, debido a las alteraciones físicas, reacciones psicológicas y sociales. Se objetivó evaluar la presencia de signos y síntomas de ansiedad y depresión y verificar asociaciones de las patologías con las variables sexo y edad con pacientes en preoperatorio de cirugías electivas. Datos recolectados en Hospital Universitario de Ribeirão Preto-SP, muestra compuesta por 100 sujetos que respondieron a la Escala de Ansiedad y Depresión Hospitalaria (HADS) y a un instrumento de datos sociodemográficos y clínicos, e informaciones relativas a las condiciones del período perioperatorio. En la evaluación de ansiedad y depresión, $40 \%$ de los individuos fueron considerados casos de ansiedad, y $22 \%$ casos de depresión. Se encontró relación estadísticamente significativa de los niveles de dichas patologías con las variables sexo masculino $(p=0,001)$, femenino $(p=0,000)$ y edad hasta 45 años $(p=0,000)$.

Descriptores: Ansiedad; Depresión; Cirugía; Atención Perioperativa.

\footnotetext{
${ }^{1}$ Enfermeira. Enfermeira da Santa Casa de Misericórdia de Monte Alto. Monte Alto, SP, Brasil. E-mail: mariana avelino@hotmail.com

2 Enfermeira, Doutora em Enfermagem Fundamental. Professora Titular da Escola de Enfermagem de Ribeirão Preto (EERP) da Universidade de São Paulo (USP). Ribeirão Preto, SP, Brasil. E-mail: rizzardo@eerp.usp.br.

${ }^{3}$ Enfermeira, Mestre em Enfermagem. Discente do Programa Interunidades de Doutoramento em Enfermagem da Escola de Enfermagem da USP e Escola de Enfermagem de Ribeirão Preto da USP. Ribeirão Preto, SP, Brasil. E-mail: luciana.paiva@usp.br.

${ }^{4}$ Enfermeira, Mestre em Enfermagem. Discente do Programa Interunidades de Doutoramento em Enfermagem da Escola de Enfermagem da USP e

Escola de Enfermagem de Ribeirão Preto da USP. Ribeirão Preto, SP, Brasil. E-mail: rsdantas@eerp.usp.br.

${ }^{5}$ Enfermeira, Doutora em Enfermagem Fundamental. Professora Associada da EERP/USP. Ribeirão Preto, SP, Brasil. E-mail: dalcala@eerp.usp.br.

${ }^{6}$ Enfermeira. Ribeirão Preto, SP, Brasil. E-mail: elainebomfim@hotmail.com.
} 


\section{INTRODUÇÃO}

O avanço das técnicas anestésicas e cirúrgicas, a evolução farmacológica de agentes anestésicos, antieméticos e analgésicos contribuíram para o crescimento contínuo dos procedimentos cirúrgicos que, aliados à hospitalização, representam uma ameaça para o doente e sua família, não só pela possibilidade de alterações físicas, mas também pelas consequentes reações psicológicas e sociais associadas à experiência da hospitalização(1-2).

O procedimento cirúrgico é frequentemente uma experiência estressante para os pacientes e familiares que requer uma série de mobilizações afetivas para lidar com a ansiedade e estresse da situação(3). Qualquer intervenção cirúrgica é considerada como uma situação crítica capaz de despertar comportamentos individuais, influenciada por múltiplos fatores emocionais, físicos, familiares, sociais, hospitalares ${ }^{(4)}$, sendo necessária a atenção psicológica dos pacientes cirúrgicos para o alcance de resultados ideais.

Inúmeros fatores podem contribuir para a ansiedade vivenciada no período anestésico-cirúrgico, dentre eles destacam-se: receio de dor, preocupação com lesões decorrentes do procedimento cirúrgico, separação da família, perda da independência, experiências anestésicas prévias, histórico de câncer, tabagismo, desordens psiquiátricas, percepção negativa do futuro, sintomas depressivos moderados a intensos e medo do diagnóstico, das complicações e da incapacidade ${ }^{(5-6)}$.

A ansiedade e a depressão podem ser medidas por escalas, inventários e questionários que podem ser de avaliação clínica ou de autoavaliação. Esses instrumentos podem facilitar a comunicação entre o médico, a equipe de enfermagem e o doente, diminuindo o sofrimento e os sintomas de ansiedade e depressão(7).

Há na literatura relatos frequentes de sintomas psicológicos de ansiedade e de depressão em pacientes no pré-operatório. Esses sintomas se combinam com as alterações da doença física, com o sofrimento psíquico e problemas sociais e dificultam a identificação dos casos de ansiedade e depressão(8).

Ansiedade pré-operatória é considerada um preditor de uma série de alterações pós-cirúrgicas incluindo aumento da dor, náuseas e vômitos e ansiedade no pósoperatório(8).

Um estudo realizado com pacientes submetidos a procedimentos cirúrgicos eletivos de pequeno e médio porte mostrou que $44,3 \%$ dos pacientes apresentaram ansiedade e $26,6 \%$ depressão, sendo que os pacientes desempregados apresentaram nível mais elevado de ansiedade $^{(9)}$.

Uma das estratégias utilizadas para minimizar os níveis de ansiedade e depressão é o fornecimento de informações sobre as condições de saúde, procedimento cirúrgico e a participação do paciente e família no autocuidado para a recuperação pós-operatória. Neste sentido, o enfermeiro deve utilizar como ferramenta a orientação pré-operatória, oferecida ao paciente em linguagem clara, respeitando seus conhecimentos e sua cultura, auxiliando na redução do medo e no esclarecimento de dúvidas(10). Esta estratégia de assistência individualizada e humanizada ao paciente pode ser realizada por meio da sistematização da assistência, pois é o meio pelo qual o enfermeiro avalia o paciente, planeja e implementa os cuidados a serem prestados, utilizando como recurso a assistência sistematizada ${ }^{(10)}$.

Os objetivos do presente estudo foram avaliar a presença de sinais e sintomas associados à presença de ansiedade e de depressão em pacientes no préoperatório de cirurgias eletivas, avaliar os níveis de ansiedade e depressão destes pacientes e verificar possíveis associações entre a presença de ansiedade ou depressão e as variáveis sexo e idade.

\section{MÉTODOS}

Estudo de delineamento descritivo e correlacional. A população consistiu de pacientes admitidos em um hospital universitário, no período de outubro de 2009 a junho de 2010, em pré-operatório de cirurgias eletivas de herniorrafia, colecistectomia, laparoscopia ou laparotomia ginecologia, gástrica ou geral. Consideraram-se como critérios de inclusão idade igual ou superior a 18 anos, saber ler e escrever, admissão para as cirurgias eletivas descritas e, como exclusão, a presença de transtornos cognitivos que impedissem a coleta de dados.

O protocolo do estudo foi aprovado pelo Comitê de Ética e Pesquisa do Hospital de Clínicas da Faculdade de Medicina de Ribeirão Preto da Universidade de São Paulo (HCFMRP-USP), sob o parecer n० 7338/2009. Todos os participantes assinaram o Termo de Consentimento Livre e Esclarecido.

Foram realizadas visitas ao Centro Cirúrgico do 
HCFMRP-USP, diariamente, para consulta da escala cirúrgica do enfermeiro. Constatado um possível participante, as pesquisadoras dirigiam-se à Enfermaria Cirúrgica, local onde o paciente se encontrava internado, para explicar sobre a importância da pesquisa e seus objetivos e convidá-lo a participar do estudo. Após concordância com a assinatura do Termo de Consentimento Livre e Esclarecido, iniciava-se a coleta de dados do período pré-operatório. Os pacientes submetidos à cirurgia ambulatorial foram entrevistados no dia da cirurgia, na recepção do centro cirúrgico.

Para coleta dos dados sociodemográficos e clínicos e informações relativas as condições do período perioperatório (doenças pré-existentes, uso de medicações, sintomatologia clínica, cirurgia realizada, tempo cirúrgico, entre outros) foi elaborado um instrumento, que foi submetido à validação de aparência e conteúdo.

Para a avaliação de ansiedade e depressão foi aplicada a Escala de Ansiedade e Depressão Hospitalar (HADS). Essa escala foi desenvolvida inicialmente para identificar sintomas de ansiedade e de depressão em pacientes de hospitais clínicos não-psiquiátricos e, posteriormente, se estendeu para outros tipos de pacientes(10-12). A HADS foi traduzida para vários idiomas e validada para o português em 1995(13). Este instrumento consiste em 14 questões - sete para ansiedade e sete para depressão, como uma escala de resposta que varia de zero a três, sendo o escore máximo para ansiedade ou depressão 21. Escores mais altos indicam presença de ansiedade e depressão. Foram adotados os pontos de corte apontados por Zigmond e Snaith para ambas as subescalas. Um escore de ansiedade maior ou igual a nove na HADS foi considerado diagnóstico de ansiedade, enquanto um escore de depressão maior ou igual a nove na HADS foi considerado diagnóstico de depressão(14).

Um ponto importante que difere a HADS das demais escalas é que, para prevenir a interferência dos distúrbios somáticos na pontuação da escala, foram excluídos os sintomas de ansiedade ou de depressão relacionados a doenças físicas, não incluindo itens como: perda de peso, anorexia, insônia, fadiga, pessimismo sobre o futuro, dor de cabeça e tontura, que podem ser sintomas de doenças físicas. Caso existam patologias que possam interferir na avaliação da presença ou ausência de transtornos de humor, os sintomas psicológicos, mais do que os sintomas somáticos, estabelecem os transtornos do humor de outras doenças clínicas $^{(8)}$.

Os dados foram processados e analisados no programa estatístico SPSS, versão 15, através de estatística descritiva e inferencial. Aplicaram-se frequências, proporções, medidas de tendência central e de variabilidade. Os escores das escalas de ansiedade e depressão foram avaliados pelas suas médias. Na comparação entre os resultados referentes às outras variáveis, foi utilizado o teste de Mann-Whitney. O nível de significância adotado foi de 0,05. Para verificar a correlação dos níveis de ansiedade e depressão foi utilizada a correlação de Spearman.

\section{RESULTADOS}

Foram incluídos no estudo 100 sujeitos que atenderam aos critérios de inclusão. Houve predominância do sexo feminino (81\%) e de baixa escolaridade, sendo que $53 \%$ dos sujeitos tinham até ensino fundamental completo e desempenhavam atividades remuneradas (52\%). A média de idade dos participantes foi de 42,4 anos. Os diagnósticos médicos mais frequentes foram: neoplasias (30\%), patologias ginecológicas (27\%), lesões do aparelho digestivo (22\%) e colorretais (21\%).

Os pacientes incluídos no estudo apresentaram uma combinação de preocupação excessiva, medo, nervosismo, receio e agitação, sendo esses sintomas mais comuns entre os pacientes que apresentaram ansiedade e depressão, como mostra a Tabela 1. 
Tabela 1: Frequência de sintomas psicológicos e somáticos segundo a presença de ansiedade e de depressão em pacientes no pré-operatório de cirurgias eletivas do HCFMRP-USP, Ribeirão Preto, 2010.

\begin{tabular}{|c|c|c|c|c|}
\hline \multirow{2}{*}{ Presença de sintomas } & \multicolumn{2}{|c|}{ Ansiedade (\%) } & \multicolumn{2}{|c|}{ Depressão(\%) } \\
\hline & S/ ansiedade & C/ ansiedade & S/ depressão & C/ depressão \\
\hline Insônia & 22,7 & 44,1 & 29,6 & 31,6 \\
\hline Agitação & 21,2 & 35,3 & 19,8 & 52,6 \\
\hline Nervosismo & 30,3 & 44,1 & 28,4 & 63,2 \\
\hline Preocupação & 42,4 & 76,5 & 44,4 & 94,7 \\
\hline Irritabilidade & 6,1 & 26,5 & 8,6 & 31,6 \\
\hline Receoso & 21,2 & 38,2 & 19,8 & 57,9 \\
\hline Respiração aumentada & 1,5 & 8,8 & 2,5 & 10,5 \\
\hline Medo & 39,4 & 73,5 & 43,2 & 84,2 \\
\hline Dor & 15,2 & 23,5 & 17,3 & 21,1 \\
\hline
\end{tabular}

No grupo estudado, no que se refere à avaliação da ansiedade obteve-se um valor médio de 6,8 pontos, em um intervalo possível de zero a 21. Quarenta por cento dos indivíduos apresentaram sintomas de ansiedade no pré-operatório. Na avaliação de depressão, obtivemos um valor médio de 4,4 pontos, sendo que $22 \%$ do grupo estudado apresentava sinais de depressão no período que antecede o procedimento cirúrgico.
Dezessete pacientes (68\%) que apresentaram ansiedade aguardaram até seis meses para realização de sua cirurgia e $36 \%$ apresentaram tempo de espera superior a um ano. Para os pacientes com depressão o tempo de espera de cirurgia foi de até seis meses (64\%) e os demais aguardaram período superior a um ano (Tabela 2).

Tabela 2: Comparação das medianas dos escores da HADS ansiedade e HADS depressão, segundo o sexo e a faixa etária em pacientes no pré-operatório de cirurgias eletivas do HCFMRP-USP, Ribeirão Preto, 2010.

\begin{tabular}{lcccc}
\hline & HADS ansiedade & HADS depressão \\
\cline { 2 - 4 } & Mediana (intervalo) & Valor $\mathbf{p}^{*}$ & Mediana (intervalo) & Valor $\mathbf{p}^{*}$ \\
\hline Sexo & $5(0-17)$ & 0,61 & 0,002 \\
$\quad$ Masculino & $7(0-20)$ & & $2(0-11)$ & \\
$\quad$ Feminino & & & $4(0-14)$ \\
Faixa etária & $7(0-20)$ & 0,552 & $3(0-14)$ & 0,272 \\
$\quad$ Até 45 anos & $6(0-13)$ & & $3(0-11)$ & \\
46 ou mais anos & & & & \\
\hline
\end{tabular}

As medidas de ansiedade e depressão foram comparadas com as variáveis que tem sido apontadas como tendo possível efeito preditor para o estado emocional como, sexo e idade ${ }^{(3,8)}$, conforme a Tabela 3.

Tabela 3. Correlação de Spearman entre as medidas de HADS ansiedade e HADS depressão, segundo sexo e faixa etária em pacientes no pré-operatório de cirurgias eletivas do HCFMRP-USP, Ribeirão Preto, 2010.

\begin{tabular}{lcc}
\hline & Variáveis & $\mathbf{r}$ \\
\hline Sexo & & $\mathbf{P}$ \\
$\quad$ Masculino & 0,709 & 0,001 \\
$\quad$ Feminino & 0,448 & 0 \\
Idade & & 0 \\
$\quad$ Até 45 anos & 0,659 & 0,273 \\
46 ou mais anos & 0,173 & \\
\hline
\end{tabular}

Os dados mostram relação estatisticamente significante dos níveis de ansiedade e depressão com as variáveis sexo masculino $(p=0,001)$, sexo feminino $(p=0,000)$ e idade até 45 anos $(p=0,000)$. A correlação da pontuação total da HADS com o sexo masculino demonstrou uma forte correlação $(r=0,709)$, sendo que os níveis de ansiedade e depressão em indivíduos mais jovens com idade até 45 anos apresentaram correlação mais forte $(r=0,659)$. Com relação ao sexo feminino esta correlação é moderada $(r=0,448)$ (Tabela 3$)$.

Não foi encontrada diferença estatisticamente significante entre a mediana dos escores de ansiedade e 
depressão e as variáveis escolaridade, tabagismo e remarcação cirúrgica.

\section{DISCUSSÃO}

Os impactos da hospitalização e dos procedimentos cirúrgicos podem levar o paciente a uma série de conflitos internos que favorecem o aumento da sua ansiedade diante do acontecimento ${ }^{(15)}$. No préoperatório frequentemente são encontrados sintomas de ansiedade e de depressão, pois neste período, os pacientes apresentam sofrimento psíquico e problemas sociais que interferem nos resultados clínicos. Além disso, estes dados muitas vezes são subnotificados por não serem reconhecidos precocemente pelos profissionais envolvidos na assistência(5).

Nesse estudo, o diagnóstico mais prevalente nos participantes foi a neoplasia (30\%), sendo mais frequentemente diagnosticada em mulheres (93\%). Diante de um diagnóstico de uma doença como o câncer, frequentemente associada à morte, a procedimentos mutiladores e sofrimento, o tratamento e a própria hospitalização podem provocar reações como raiva, mágoa, tristeza, dificuldade para dormir e se alimentar, ansiedade e depressão. Entre os sintomas mais frequentes estão, ainda, a preocupação, medo, nervosismo, receio e agitação. A prevalência destas perturbações psicológicas em doentes oncológicos é mais elevada devido à convivência direta com a dor, com o desfiguramento, com a separação e com a morte(16).

A ansiedade é um estado emocional que guarda relação com o medo ${ }^{(7)}$. Este estado caracteriza-se por um conjunto de respostas representativas do estado ansioso como: elevação da frequência cardíaca, alterações na respiração e na pressão sanguínea, sudorese, tremores e sensações de falta de ar e inquietação. Dependendo da intensidade destes sintomas poderá ser considerada normal ou patológica(7).

Pesquisas anteriores já haviam mostrado que a ansiedade pré-operatória mais prevalente em mulheres ${ }^{(3,8)}$; um resultado que não coincide com os encontrados nesse estudo e que pode estar relacionado aos mecanismos de enfrentamento ou adaptação que os pacientes do sexo masculino apresentaram diante da intervenção cirúrgica.

Ao analisarmos a idade verificamos que os pacientes mais jovens apresentaram maior frequência de ansiedade. No momento da internação hospitalar, os pacientes vivenciam perdas financeiras, afastamento das relações sociais e medo do diagnóstico, das complicações e da incapacidade ${ }^{(1,5-7)}$. Para os mais jovens pode haver uma sensação de perda exagerada quanto mais intensa forem as atividades realizadas no dia a dia.

No grupo estudado, $40 \%$ dos indivíduos foram considerados casos de ansiedade. Essa frequência de ansiedade mostrou que uma parcela considerável de pacientes apresenta esses sintomas e, portanto, requerem uma avaliação mais detalhada sobre seu estado mental no período pré-operatório.

O tempo de espera pela cirurgia e, ainda, a suspensão e a remarcação da cirurgia podem contribuir para aumentar o medo e o sentimento de desconfiança, diante de um resultado duvidoso; porém, em nosso estudo, esses aspectos não foram associados aos níveis de ansiedade e depressão. A cada cirurgia e a cada reinternação, o individuo vivencia um novo evento, mesmo nos casos em que o paciente já conhece os procedimentos técnicos ${ }^{(5)}$.

A comunicação efetiva entre o paciente e a equipe multiprofissional é de vital importância para que se obtenham melhores resultados no pós-operatório, haja vista a complexidade do procedimento, o uso de altas tecnologias, os cuidados requeridos e a associação com as crenças que envolvem a cirurgia ${ }^{(11)}$.

Durante a visita pré-operatória a equipe multidisciplinar deve fornecer informações técnicas específicas nas distintas modalidades cirúrgicas, considerando suas peculiaridades e os inúmeros fatores que diferenciam os protocolos e procedimentos ${ }^{(14)}$. Nesse sentido, é importante que o enfermeiro, durante a visita pré-operatória, forneça informações que contemplem todas as ações a serem desenvolvidas no período perioperatório, reduzindo assim, o nível de ansiedade da paciente, por meio da abordagem de sentimentos como o medo, a apreensão e preocupação relacionados à falta de informações e cuidados adequados a estas pacientes.

As orientações de enfermagem devem enfocar desde as formas de prevenção de uma nova patologia, as formas de seguimento do tratamento vigente, a importância da adesão à terapêutica indicada, as condutas apropriadas a cada fase do processo saúdedoença vivenciado, o esclarecimento das dúvidas acerca desse processo complexo e o incentivo ao autocuidado $^{(1)}$. 


\section{CONCLUSÕES}

Este estudo confirmou a presença de ansiedade e depressão no pré-operatório de cirurgias eletivas, com associação estatisticamente significante com as variáveis sexo e idade. Esses dados demonstram a necessidade da incorporação de novas estratégias de assistência que se preocupem com as dimensões psicossociais dos pacientes, permitindo com isso o diagnóstico precoce e tratamento dos distúrbios de ansiedade e depressão.

\section{REFERÊNCIAS}

1. Carvalho ARS, Matsuda LM, Stuch RAG, Coimbra, JAH. Investigando as orientações oferecidas ao paciente em pósoperatório de revascularização miocárdica. Rev. Eletr. Enf. [Internet]. 2008 [cited 2012 jun 18];10(2):504-12. Available from: http://www.fen.ufg.br/revista/v10/n2/v10n2a21.htm. 2. Rhodes L, Miles G, Pearson A. Patient subjective experience and satisfaction during the perioperative period in the day surgery setting: A systematic review. Int J Nurs Pract. 2006;12:176-192. 3. Santos AF, Santos LA, Melo DO, Alves AJ. Estresse pré-operatório: comparação entre pacientes do SUS e conveniados. Psicologia: Reflexão e Crítica. 2009;22(2):269-276.

4. Daian MR, Petroianu A, Albeti LR, Jeunon EE. Estresse em cirurgia de grande porte. Rev. méd. Minas Gerais. 2010;20(4):490-499. 5. Alves MLM, Pimentel AJ, Guaratini AA, Marcolino JAM, Gozzani JL, Mathias LAST. Ansiedade no período pré-operatório de cirurgias de mama: estudo comparativo entre pacientes com suspeita de câncer e a serem submetidas a procedimentos cirúrgicos estéticos. Rev Bras Anestesiol. 2007;57:147-1565.

6. Magalhães Filho LL, Segurado A, Marcolino JAM, Mathias LAST. Impacto da avaliação pré-anestésica sobre a ansiedade e depressão dos pacientes cirúrgicos com câncer. Rev Bras Anestesiol. 2006;56:126-136.

7. Netto R, Mestre M, Santos DCLR, Zotto LLS. Ansiedade e depressão em pacientes com tumores do sistema nervoso, hospitalizados à espera da cirurgia. Rev. bras. de ter. comp. cogn. 2009;11(2):267-284.

8. Caumo W, Schmidt AP, Schneider CN, Bergmann J, Iwamoto CW, Adamatti LC, et al. Risk factors for postoperative anxiety in adults. Anaesthesia. 2001;56:720-728.

9. Marcolino JAM, Mathias LAST, Filho LP, Guaratini AA, Suzuki FM, Alli LAC. Escala hospitalar de ansiedade e depressão: Estudo da validade de critério e da confiabilidade com pacientes no préoperatório. Rev Bras Anestesiol. 2007;57(1):52-62.

10. Prá LA, Piccoli M. Enfermagem perioperatória: diagnósticos de enfermagem fundamentados na teoria de Ida Jean Orlando. Rev. Eletr. Enf. [Internet]. 2004 [cited 2012 jun 18];10(2): 6(2):234-253. Available from: http://www.fen.ufg.br/fen revista/revista6 2/enfer.html. 11. Santos J, Henckmeier L, Benedet AS. O impacto da orientação pré-operatória na recuperação do paciente cirúrgico. Enfermagem em Foco. 2011;2(3):184-187.

12. Andrews B, Hejdenberg J, Wilding J. Student anxiety and depression: Comparison of questionnaire and interview assessments. J. Affect Disord. 2006;95:29-34

13. Botega NJ, Bio MR, Zomignani MA, Garcia CJ, Pereira WAB. Transtornos de humor em enfermarias de clínica médica e validação de escala de medida (HAD) de ansiedade e depressão. Rev Saude Publica. 1995;29:355-363.

14. Zigmond AS, Snaith RP. The hospital anxiety and depression scale. Acta Psychiatrica Scandinavica. 1983;67:361-370.

15. Costa VASF, Silva SCF, Lima VCP. O pré-operatório e a ansiedade do paciente: a aliança entre o enfermeiro e o psicólogo. Rev. SBPH. 2010;13(2):282-298.

16. Mitchell M. Psychological care of patients undergoing elective surgery. Nursing Standard. 2007;12:48-55. 